\title{
ESTOQUE DE CARBONO ORGÂNICO NO SOLO E EMISSÕES DE DIÓXIDO DE CARBONO INFLUENCIADAS POR SISTEMAS DE MANEJO NO SUL DO BRASIL ${ }^{(1)}$
}

\author{
Falberni de Souza Costa ${ }^{(2)}$, Cimélio Bayer ${ }^{(3)}$, Josiléia Acordi \\ Zanatta $^{(4)}$ \& João Mielniczuk ${ }^{(3)}$
}

\begin{abstract}
RESUMO
O C fotossintetizado adicionado ao solo pelos resíduos vegetais (C-resíduo), as emissões de $\mathrm{C}$ na forma de dióxido de carbono $\left(\mathrm{C}-\mathrm{CO}_{2}\right)$ e o estoque de $\mathrm{C}$ orgânico do solo (C-solo) são componentes do ciclo deste elemento no sistema solo-plantaatmosfera. O efeito de práticas de manejo de solo sobre esses componentes necessita de melhor entendimento, visando à identificação de um sistema com potencial de reter $\mathrm{C}$ atmosférico no solo e contribuir para a mitigação do aquecimento global. Neste estudo, as emissões de $\mathrm{C}-\mathrm{CO}_{2}$, o estoque de $\mathrm{C}$ e as adições de $\mathrm{C}$ pelos resíduos vegetais foram avaliados em experimento de longa duração (18 anos), localizado em Eldorado do Sul (RS). O quociente C-CO ${ }_{2} /(\mathrm{C}-$ resíduo + $\mathrm{C}$-solo) foi proposto como índice da capacidade de sistemas de preparo e de cultura em conservar $\mathrm{C}$ no solo (ICC). A emissão de $\mathrm{C}-\mathrm{CO}_{2}$ foi medida durante 17 meses; a quantidade de C-resíduo foi estimada com base em amostragens da massa de plantas de cobertura de inverno e no índice de colheita do milho; e o estoque de C-solo, avaliado na camada de 0-0,2 m do solo submetido aos sistemas de preparo convencional $(\mathrm{PC})$ e plantio direto $(\mathrm{PD})$, associados às sucessões de aveia (Avena strigosa Schreb)/milho (Zea mays $\mathrm{L}_{\text {.) }}$ (A/M) e ervilhaca comum (Vicia sativa $\mathrm{L}_{\text {. }}$ )/ milho (E/M). Com objetivo de avaliar a relação das emissões de $\mathrm{C}-\mathrm{CO}_{2}$ com fatores ambientais, foram monitoradas a temperatura a $0,05 \mathrm{~m}$ de profundidade e a umidade gravimétrica do solo nas camadas de 0-0,05; 0,05-0,1; e 0,1-0,2 m. Em comparação ao estoque de C-solo no início do experimento $\left(33,4 \mathrm{tha}^{-1}\right)$, o balanço foi negativo no solo em PC (-0,31 $\mathrm{t} \mathrm{ha}^{-1}$ ano $^{-1}$ no $\mathrm{A} / \mathrm{M}$ e $-0,10 \mathrm{t} \mathrm{ha}^{-1} \mathrm{ano}^{-1}$ no E/M) e positivo no solo em $\mathrm{PD}\left(0,15 \mathrm{t} \mathrm{ha}^{-1}\right.$ ano $\left.^{-1}\right)$ apenas quando associado ao sistema $\mathrm{E} / \mathrm{M}$, o qual apresentou maior aporte de resíduos. As taxas mensais médias das emissões variaram entre $0,27 \mathrm{~g} \mathrm{~m}^{-2}$ de $\mathrm{C}-\mathrm{CO}_{2}$ no inverno (média das temperaturas mínimas $=8{ }^{\circ} \mathrm{C}$ ) e $1,36 \mathrm{~g} \mathrm{~m}^{-2}$
\end{abstract}

\footnotetext{
(1) Parte da pesquisa de Tese de Doutorado do primeiro autor apresentada ao Programa de Pós-Graduação em Ciência do Solo (PPGCS) da Universidade Federal do Rio Grande do Sul - UFRGS. Recebido para publicação em novembro de 2006 e aprovado em outubro de 2007.

(2) Professor do Campus Floresta, Universidade Federal do Acre - UFAC. Estrada do Canela Fina, km12, Colônia São Francisco, CEP 69980-000 Cruzeiro do Sul (AC). E-mail: falberni@ufac.br

(3) Professor do Departamento de Solos, Universidade Federal do Rio Grande do Sul - UFRGS. Av. Bento Gonçalves 7712, Caixa Postal 15100, CEP 91501-970 Porto Alegre (RS). Bolsista do CNPq. E-mail: cimelio.bayer@ufrgs.br

${ }^{(4)}$ Doutoranda do PPGCS-UFRGS. E-mail: josizanatta@yahoo.com.br
} 
de $\mathrm{C}-\mathrm{CO}_{2}$ no verão (média das temperaturas máximas $=38^{\circ} \mathrm{C}$ ), que se relacionaram com a temperatura do solo $(r>0,85)$. A emissão total de $\mathrm{C}-\mathrm{CO}_{2}$ no período variou entre 3,6 e 4,0 t ha $^{-1}$ de $\mathrm{C}-\mathrm{CO}_{2}$, não tendo sido verificada diferença significativa entre os sistemas de preparo de solo e de cultura. Entretanto, o ICC evidenciou que o potencial dos sistemas de manejo em conservar $\mathrm{C}$ no solo aumentou na ordem PC A/M < PC E/M < PD A/M < PD E/M. As condições menos oxidativas contribuíram para o balanço positivo de $\mathrm{C}$ no solo em plantio direto, característica que é potencializada pela utilização de sistemas de cultura com leguminosas como plantas de cobertura, que favorecem o acúmulo de $\mathrm{C}$ no solo por permitirem maior produção de massa das gramíneas cultivadas em sucessão, devido ao fornecimento de $\mathbf{N}$.

Termos de indexação: dióxido de carbono, carbono orgânico, plantio direto, plantas de cobertura, leguminosas.

\title{
SUMMARY: CARBON STOCK AND CARBON DIOXIDE EMISSIONS AS AFFECTED BY SOIL MANAGEMENT SYSTEMS IN SOUTHERN BRAZIL
}

\begin{abstract}
Carbon $(C)$ addition through crop residues (residue- $C$ ), $C$ dioxide emission $\left(\mathrm{CO}_{2}-\mathrm{C}\right)$ and the soil $C$ stock (soil-C) are components of the $C$ cycle in the soil-plant-atmosphere system. This 18-year study was conducted to identify agricultural practices that could potentially increase $C$ retention in the soil and lessen global warming trends. The three $C$ pools (residue- $\mathrm{C}, \mathrm{CO}_{2}$ - $\mathrm{C}$ and soil-C) under different tillage systems (CT-conventional tillage and NT-no tillage) and cropping systems (O/M-oat (Avena strigosa Schreb)/maize (Zea mays $L$.) and $V / M$-vetch (Vicia sativa $L$.)/maize)) were evaluated and the $\mathrm{CO}_{2}$ - $\mathrm{C} /[$ residue$C+$ soil-C] quotient was proposed as $C$ retention index (CRI), where low values indicate a high capacity of the management system to keep $\mathrm{C}$ in the soil. The $\mathrm{CO}_{2}$ - $\mathrm{C}$ emissions were measured for 17 months (between November 2002 and March 2004), sampling of aboveground residues of cover crops and harvest indexes of maize were used to quantify $C$ addition by cropping systems, and soil-C stocks $(0-0.2 \mathrm{~m})$ were evaluated in 2003 . The soil temperature $(0.05 \mathrm{~m})$ and gravimetric water content $(0-0.05,0.05-0.1$ and $0.1-0.2 \mathrm{~m})$ were also monitored from May 2003 onwards. In comparison to 1985, the $C$ balance was negative in the soil under CT (-0.31 $t \mathrm{ha}^{-1} \mathrm{yr}^{-1}$ for $\mathrm{O} / \mathrm{M}$ and $-0.10 t \mathrm{ha}^{-1} \mathrm{yr}^{-1}$ for V/M). On the other hand, the $C$ balance was positive in NT soil, but only under V/M $\left(+0.15 t \mathrm{ha}^{-1} \mathrm{yr}^{-1}\right)$ due to the greater $\mathrm{C}$ addition by crop residues. The $\mathrm{CO}_{2}-\mathrm{C}$ emission was related to the soil

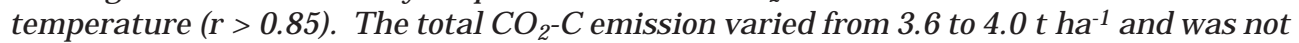
affected by the soil management systems. However, the CRI allowed a clear discrimination of the soil management systems to keep $C$ in the soil. The $C$ retention potential increased in the following order: $C T O / M<C T V / M<N T O / M<N T V / M$. Results reinforced the importance of symbiotically-fixed $N$ by legume crops for $C$ accumulation in untilled soils due to greater biomass production by succeeding graminaceae.
\end{abstract}

Index terms: carbon dioxide, cover crops, legumes, no-tillage, soil organic carbon.

\section{INTRODUÇÃO}

No âmbito das mudanças climáticas globais, o solo e suas formas de uso estão em foco, sobretudo no que se refere à agricultura. Os solos agrícolas podem atuar como dreno ou fonte de gases de efeito estufa (GEE), dependendo do sistema de manejo a que forem submetidos (IPCC, 2001). Sistemas de manejo que aumentem a adição de resíduos vegetais e a retenção de $\mathrm{C}$ no solo se constituem em alternativas importantes para aumentar a capacidade de dreno de C-CO $\mathrm{CO}_{2}$ atmosférico e mitigação do aquecimento global (Amado et al., 2001; Bayer et al., 2006).
Várias são as ênfases e as formas de quantificar o efeito de sistemas de manejo sobre os fluxos de $\mathrm{C}$ no sistema solo-planta-atmosfera citadas na literatura (Paustian et al., 1997; Janzen et al., 1998). O balanço de $\mathrm{C}$ no solo é dependente da relação entre as adições de $\mathrm{C}$ fotossintetizado pelas plantas (parte aérea e raízes) e as perdas de $\mathrm{C}$ para a atmosfera resultantes da oxidação microbiana do $\mathrm{C}$ orgânico a $\mathrm{CO}_{2}$. A magnitude desses processos pode ser avaliada em experimentos de longa duração, pela quantificação dos estoques de C orgânico no solo, em comparação ao estoque inicial de C-solo (Costa et al., 2006). 
Embora o método de avaliação do balanço de $\mathrm{C}$ no solo quantifique os fluxos anuais médios de $\mathrm{C}$ em sistemas de manejo, ele não é adequado na avaliação da dinâmica temporal da emissão de $\mathrm{C}-\mathrm{CO}_{2}$ em função das estações do ano e de alterações nos fluxos de $\mathrm{C}-\mathrm{CO}_{2}$ devido a fatores ambientais e de práticas de manejo de solo (Franzluebbers et al., 1995). O preparo de solo e o manejo de culturas afetam as taxas metabólicas dos microrganismos nos processos de decomposição dos resíduos vegetais e da matéria orgânica no solo (MOS), as quais também são influenciadas pela temperatura e umidade do solo (La Scala Jr. et al., 2006). Geralmente, a atividade decompositora dos microrganismos atinge valores elevados no período imediatamente posterior ao revolvimento do solo, o qual é associado às estações de temperatura mais elevada no Sul do Brasil, decrescendo posteriormente à atividade basal, devido à redução ao mínimo das frações orgânicas de fácil decomposição (Paul \& Clark, 1996; La Scala Jr. et al., 2006).

A atividade basal e a emissão de $\mathrm{C}-\mathrm{CO}_{2}$ decorrente da ação decompositora dos microrganismos heterotróficos no solo são dependentes das condições de solo, principalmente do conteúdo de MOS e da disponibilidade de resíduos vegetais, os quais se constituem nas principais fontes de $\mathrm{C}$ à microbiota $\mathrm{e}$ têm influência nas propriedades físicas, químicas e biológicas do solo (Vargas \& Scholles, 2000; Costa et al., 2003; Ciotta et al., 2004). Nesse contexto, a emissão basal de C-CO $\mathrm{CO}_{2}$ será diferente em sistemas de manejo que apresentem distintas adições de resíduos vegetais e estoques de $\mathrm{C}$-solo, determinando que o uso isolado das emissões de $\mathrm{C}-\mathrm{CO}_{2}$ não seja indicador adequado do potencial de sistemas de manejo na conservação de $\mathrm{C}$ no solo, devendo-se considerar para isso as emissões de $\mathrm{C}-\mathrm{CO}_{2}$ por unidade de $\mathrm{C}$ orgânico na MOS e em resíduos vegetais. Dessa forma, sistemas de manejo com menor emissão de $\mathrm{C}-\mathrm{CO}_{2}$ por unidade de $\mathrm{C}$ na MOS e em resíduos vegetais apresentam maior potencial de conservação de $\mathrm{C}$ no solo, em comparação com sistemas de maior emissão de $\mathrm{C}-\mathrm{CO}_{2}$ por unidade de $\mathrm{C}$ potencialmente disponível para oxidação microbiana.

O objetivo deste estudo foi avaliar o potencial de sistemas de preparo de solo e de culturas na conservação de C em um Argissolo Vermelho da Depressão Central do Rio Grande do Sul (RS). Além do balanço de $\mathrm{C}$ no solo nos diferentes sistemas de manejo, avaliou-se a adequação do quociente $\mathrm{C}-\mathrm{CO}_{2} /$ [C-resíduo+C-solo] como um índice da capacidade de sistemas de manejo em conservar $\mathrm{C}$ no solo.

\section{MATERIAL E MÉTODOS}

\section{Caracterização edafoclimática}

O estudo foi feito na região da Depressão Central do $\mathrm{RS}$, em experimento realizado na Estação
Experimental Agronômica da Universidade Federal do Rio Grande do Sul - UFRGS (30 $05^{\prime} 27$ " S; $51^{\circ}$ 40 ' 18 " W), município de Eldorado do Sul, a $46 \mathrm{~m}$ de altitude. O clima da região é subtropical úmido e Cfa, segundo classificação climática de Köppen. As médias anuais da temperatura do ar e da precipitação pluvial são, respectivamente, de $19,2^{\circ} \mathrm{C}$ e $1.446 \mathrm{~mm}$, com médias mensais da temperatura variando de $9{ }^{\circ} \mathrm{C}$ no inverno a $25{ }^{\circ} \mathrm{C}$ no verão (Bergamaschi et al., 2003). O solo da área experimental é um Argissolo Vermelho distrófico típico (Embrapa, 1999), com $540 \mathrm{~g} \mathrm{~kg}^{-1} \mathrm{de}$ areia, 240 de silte e 220 de argila.

\section{Experimento de longa duração}

O experimento foi instalado em 1985, em uma área com avançado grau de degradação física, sendo composto por três sistemas de preparo de solo (PCpreparo convencional, PR-preparo reduzido e PDplantio direto) nas parcelas principais ( $15 \times 20 \mathrm{~m})$, três sistemas de culturas (A/M-aveia-preta [(Avena strigosa Schreb)/milho (Zea mays L.), E/M-ervilhaca comum (Vicia sativa L.)/milho e A+E/M+C-aveia+ervilhaca comum/milho+caupi (Vigna unguiculata L.)] nas subparcelas $(5 \times 20 \mathrm{~m})$ e duas doses de $\mathrm{N}$ mineral (uréia) na cultura do milho (0 e $180 \mathrm{~kg} \mathrm{ha}^{-1}$ de N), aplicado em faixas $(45 \times 10 \mathrm{~m})$ nos blocos. Os tratamentos foram dispostos segundo delineamento de blocos casualizados, com três repetições. Neste estudo, foram avaliados somente os tratamentos PC e $\mathrm{PD}$, ambos nos sistemas A/M e E/M e sem aplicação de $\mathrm{N}$ mineral.

Os preparos de solo foram realizados somente na primavera/verão, sendo as culturas de inverno semeadas ( $80 \mathrm{~kg} \mathrm{ha}^{-1}$ de semente) em plantio direto nos meses de abril/maio. No PC, o preparo de solo consistiu em uma aração e duas gradagens na primavera, anterior à semeadura do milho, enquanto no PD o manejo das culturas de inverno consistiu na aplicação de herbicida dessecante à base de glifosate (720 $\mathrm{g} \mathrm{ha}^{-1}$ de IA) e passagem de rolo-faca. O milho foi semeado em setembro-outubro, com população aproximada de 50-60 mil plantas ha ${ }^{-1}$. Detalhes da condução experimental são descritos em Bayer et al. (2000) e Lovato et al. (2004).

\section{Avaliação da adição de $\mathrm{C}$ pelas culturas de cobertura e milho}

$\mathrm{A}$ adição de $\mathrm{C}$ pelas culturas de cobertura ao solo foi avaliada a partir de amostragem, no período de pleno florescimento, da fitomassa (método do quadrado de $1 \mathrm{~m}^{2}$ ), a qual foi seca em estufa até peso constante e quantificada em relação a sua massa de resíduos secos. As adições de C pelo milho foram estimadas a partir da relação entre a produtividade da cultura nos diferentes sistemas de manejo e a produção de MS da parte aérea do milho [equação 1, obtida por Lovato (2001)] para esta mesma área experimental. Considerou-se o percentual de $40 \%$ de $\mathrm{C}$ na matéria seca (MS) das plantas de cobertura e do milho (Aita et 
al., 1994; Bayer et al., 1998; Spagnollo et al., 2002) e a contribuição do sistema radicular na adição de $\mathrm{C}$ equivalente a $30 \%$ do $\mathrm{C}$ adicionado pela parte aérea das culturas (Fehrenbacher \& Alexander, 1955; Buyanovsky \& Wagner, 1986; Balesdent \& Balabane, 1992; Crozier \& King, 1993; Bolinder et al., 1997; Kissele et al., 2001).

$$
\begin{gathered}
\mathrm{MS}_{\text {adicionada }}\left(\mathrm{t} \mathrm{ha}^{-1}\right)=[0,96 * \text { rendimento de grãos } \\
\left.\left(\mathrm{t} \mathrm{ha}^{-1}\right)+2,91\right]
\end{gathered}
$$

\section{Amostragem do solo, análise dos teores de C orgânico e cálculo de seus estoques no solo}

O solo foi amostrado em outubro de 2003 nas camadas de $0-0,025,0,025-0,05,0,05-0,1$ e $0,1-0,2 \mathrm{~m}$, seco, moído a 0,15 mm em gral de ágatha, e analisado quanto ao teor de $\mathrm{C}$ orgânico por combustão seca, em analisador Shimadzu - TOC (modelo $\mathrm{V}_{\mathrm{CSH}}$ ). A partir dos valores de densidade de solo obtidos por Silva et al. (2005), foram calculados os estoques de C orgânico da camada de $0-0,2 \mathrm{~m}$ em massa equivalente de solo (Ellert \& Bettany, 1995), utilizando-se como referência a massa de solo $\left(44,7 \mathrm{t} \mathrm{ha}^{-1}\right)$ de uma área de campo nativo adjacente ao experimento (Zanatta et al., 2007). O estoque de C-solo no início do experimento (em 1985), o qual havia sido analisado por oxidação com dicromato de potássio e aquecimento externo, foi corrigido a partir da relação entre os teores de $\mathrm{C}$ obtidos por esse método e o utilizado no presente estudo. Para isso, amostras de solo com ampla variação nos teores de C orgânico foram selecionadas e analisadas quanto ao teor de $\mathrm{C}$ orgânico pelos dois métodos, gerando um fator de correção para o estoque de $\mathrm{C}$ orgânico do início do experimento (Zanatta et al., 2007).

\section{Avaliação das emissões de carbono na forma de dióxido de $\mathrm{C}\left(\mathrm{C}-\mathrm{CO}_{2}\right)$ do solo}

As emissões de $\mathrm{C}_{-} \mathrm{CO}_{2}$ foram avaliadas pelo método da "captura" do C-CO $\mathrm{CO}_{2}$ em solução de hidróxido de sódio (Anderson, 1982), de novembro de 2002 a março de 2004 (17 meses), totalizando 33 avaliações. A freqüência das avaliações foi estabelecida de acordo com a estação do ano e com as práticas de manejo, sendo diária após o manejo das culturas de inverno e o preparo do solo na primavera, e uma vez por mês em dezembro de 2002 , julho a setembro de 2003 e nos meses avaliados em 2004. Nos demais meses, foram realizadas no mínimo duas avaliações. Em abril de 2003 não houve avaliação, sendo a emissão de $\mathrm{C}-\mathrm{CO}_{2}$ estimada por interpolação entre as emissões nos meses de março e maio.

O sistema de coleta consistiu de uma base metálica, com canal de $0,07 \mathrm{~m}$ de profundidade, sobre o qual foi disposta uma câmara cilíndrica de policloreto de vinila (PVC) de 0,2 e 0,25 m de altura e diâmetro, respectivamente, cuja extremidade superior foi fechada por uma tampa também de PVC. A base foi inserida no solo $(0,05 \mathrm{~m})$, nas entrelinhas das culturas da estação anterior, e permaneceu fixa ao longo de cada estação de cultivo. Na extremidade inferior do cilindro foi colada uma borracha para auxiliar a vedação do sistema, a qual foi realizada pela colocação de água dentro do canal da base, além do uso de molas para fixar a câmara à base metálica. Na tampa da câmara foi perfurado um orifício de aproximadamente $10 \mathrm{~mm}$ de diâmetro, o qual teve a função de equilibrar a pressão interna da câmara com a pressão atmosférica quando da disposição da câmara sobre a base, após o que o orifício foi vedado por septo de borracha. Utilizaram-se três câmaras por tratamento, em cada uma das três repetições do experimento.

As coletas foram realizadas em duas microparcelas de $2 \times 2 \mathrm{~m}$, as quais foram dispostas nas extremidades das subparcelas $(5 \times 20 \mathrm{~m})$ do experimento e foram utilizadas alternadamente. A alternância do uso das microparcelas possibilitou que durante todo o período de avaliações uma das microparcelas fosse mantida com plantas em crescimento (aveia, vica ou milho), enquanto a outra fosse avaliada sem plantas, mas com resíduos vegetais sobre o solo, visando somente avaliar a emissão resultante do solo e dos resíduos, excluindo a contribuição da respiração das raízes. Por exemplo, no inverno uma microparcela tinha as culturas de cobertura em crescimento, e a outra foi utilizada na avaliação da emissão $\mathrm{C}-\mathrm{CO}_{2}$. Posteriormente ao manejo das culturas de inverno, o uso das microparcelas foi invertido e a emissão de $\mathrm{C}-\mathrm{CO}_{2}$ foi avaliada na microparcela sobre os resíduos das culturas de inverno, enquanto a outra microparcela recebeu a cultura do milho. É importante salientar que as microparcelas recebiam resíduos das culturas da estação após o final da avaliação das emissões de $\mathrm{C}-\mathrm{CO}_{2}$.

Taxas mensais de emissão de $\mathrm{C}-\mathrm{CO}_{2}$ foram calculadas pelo quociente entre a área sob a curva da emissão de $\mathrm{C}-\mathrm{CO}_{2}$ de cada mês (método do trapézio) e o respectivo número de dias do mês. A emissão de C- $\mathrm{CO}_{2}$ total nos 17 meses foi calculada por integração da área sob a curva de efluxo de $\mathrm{C}-\mathrm{CO}_{2}$ em cada sistema de manejo do solo.

\section{Monitoramento da temperatura e da umidade do solo}

As avaliações da temperatura e da umidade do solo foram iniciadas a partir da $11^{\text {a }}$ avaliação (maio de 2003). O registro da temperatura (a $0,05 \mathrm{~m}$ de profundidade) foi às 9 e $15 \mathrm{~h}$ do dia da avaliação da emissão de $\mathrm{C}-\mathrm{CO}_{2}$, e às $9 \mathrm{~h}$ do dia seguinte. Foram utilizados termômetros de mercúrio, instalados nas microparcelas de dois blocos do experimento. Temperaturas diárias médias foram calculadas a partir das temperaturas mínimas (9 h do primeiro e segundo dia de avaliação) e da temperatura máxima $(15 \mathrm{~h})$. A cada avaliação da emissão de $\mathrm{C}-\mathrm{CO}_{2}$, o solo foi amostrado com trado $(0-0,05,0,05-0,1$ e $0,1-0,2 \mathrm{~m})$, para determinação da umidade gravimétrica. 
Estimativa do potencial dos sistemas de manejo em conservar $\mathrm{C}$ no solo

O potencial de um sistema de manejo em conservar $\mathrm{C}$ no solo foi avaliado pela emissão de $\mathrm{C}-\mathrm{CO}_{2}$ por unidade de $\mathrm{C}$ nos resíduos vegetais e no solo $(0-0,2 \mathrm{~m})$ (equação 2), expresso como índice de conservação de $\mathrm{C}$ no solo (ICC), na unidade de g C-CO 2 kg C (resíduo + solo $)^{-1}$.

$$
I C C=\frac{\mathrm{C}-\mathrm{CO}_{2}}{C-\text { residuo }+\mathrm{C}-\text { solo }}
$$

\section{Informações meteorológicas}

Os dados de temperatura do ar, radiação solar e precipitação pluvial foram obtidos no Departamento de Plantas Forrageiras e Agrometeorologia (UFRGS), cuja estação meteorológica está situada também na Estação Experimental Agronômica da UFRGS.

\section{Análise estatística}

Os dados de estoque de $\mathrm{C}$ orgânico, a temperatura e a umidade do solo foram submetidos à análise de variância por data de avaliação, e as médias, comparadas pelo teste de Duncan a $5 \%$. A umidade do solo foi analisada por camada. A análise descritiva foi utilizada para adições de C-resíduo e emissões de $\mathrm{C}-\mathrm{CO}_{2}$. As relações das emissões de $\mathrm{C}-\mathrm{CO}_{2}$ com a temperatura e umidade do solo foram analisadas por correlação.

\section{RESULTADOS E DISCUSSÃO}

\section{Condições meteorológicas durante o período de estudo}

As variações da temperatura média do ar no período avaliado(11/2002 a 3/2004) foram características para a região, com temperaturas mínimas ocorrendo no inverno $\left(13^{\circ} \mathrm{C}\right)$, intermediárias no outono e primavera $\left(18^{\circ} \mathrm{C}\right)$ e máximas no verão $\left(24^{\circ} \mathrm{C}\right)$. Já as variações da precipitação pluvial no período foram irregulares (Figura 1a), apresentando tendência de aumento na primavera, sendo dezembro geralmente o mês mais chuvoso. Entre os anos, o verão de 2003 teve maior volume de chuva em relação a 2004 (Figura 1a). Destaque é dado a fevereiro e outubro de 2003, meses nos quais choveu 197 e $277 \mathrm{~mm}$, respectivamente, correspondendo a precipitação ocorrida nesses dois meses a aproximadamente um terço da precipitação anual total.

\section{Adição de C-resíduo e balanço de C orgânico no solo}

A adição de C-resíduo no período de avaliação variou de 5,8 a 7,5 t ha-1, sendo maior no sistema E/M (6,5 no $\mathrm{PC}$ e 7,5 t ha-1 no $\mathrm{PD}$ ) do que no sistema $\mathrm{A} / \mathrm{M}$ (5,8 t ha-1 no PD e no PC). Como todos os tratamentos avaliados não receberam aplicação de fertilizantes nitrogenados, em grande parte a variação das adições de $\mathrm{C}$ nos sistemas de cultura foi devido ao diferente crescimento da cultura do milho em sucessão à ervilhaca comum $(\mathrm{E} / \mathrm{M})$ e à aveia-preta $(\mathrm{A} / \mathrm{M})$.

A adição diferenciada de resíduos vegetais ao solo, durante os 18 anos de condução do experimento, afetou os estoques de $\mathrm{C}$ orgânico no solo, os quais variaram de $27,8 \mathrm{t} \mathrm{ha}^{-1}$ (PC A/M) a 36,1 $\mathrm{t} \mathrm{ha}^{-1}$ (PD E/M). O balanço de $\mathrm{C}$ no solo foi sempre negativo sob PC (-0,31 t ha-1 ano-1 no A/M e -0,10 t ha ${ }^{-1}$ ano $^{-1}$ no E/M), evidenciando que o solo cultivado neste sistema de preparo atuou como uma fonte de $\mathrm{C}-\mathrm{CO}_{2}$ para a atmosfera, independentemente do sistema de cultura adotado. Em PD, o solo apresentou influxo líquido de $\mathrm{C}\left(0,15 \mathrm{t} \mathrm{ha}^{-1} \mathrm{ano}^{-1}\right)$ somente no sistema $\mathrm{E} / \mathrm{M}$, o qual apresentou maior adição de resíduos ao solo, principalmente pela cultura do milho em sucessão. No sistema A/M, em que a adição de resíduos vegetais foi menor, o solo em PD também atuou como fonte de $\mathrm{C}-\mathrm{CO}_{2}$ para a atmosfera numa taxa de $0,12 \mathrm{t} \mathrm{ha}^{-1}$ ano $^{-1}$ (Quadro 1). Esse fato resultou da baixa capacidade de aporte de $\mathrm{C}$ do sistema $\mathrm{A} / \mathrm{M}$, cuja adição anual foi inferior à necessidade do sistema de $\mathrm{PD}$ para contrabalancear a oxidação do material orgânico pela microbiota do solo, ocorrendo diminuição do estoque de $\mathrm{C}$ do solo ao longo do tempo mesmo cultivado em PD. Outros estudos nesta mesma área experimental evidenciaram a necessidade de 13 e $21 \mathrm{t} \mathrm{ha}^{-1}$ de MS no PD e PC, respectivamente, para manter o estoque inicial de C no solo (Zanatta et al., 2007).

Os resultados ressaltam a importância do alto aporte de resíduos vegetais mesmo no sistema PD quando se visa ao acúmulo de C orgânico no solo, similarmente ao que já foi observado por Lovato et al. (2004) e Bayer et al. (2006). Nesse sentido, Drinkwater et al. (1998) e Amado et al. (2001) sugerem que o uso de leguminosas, combinado com maior diversidade de espécies em sucessão ou rotação de culturas, aumenta de forma significativa a retenção de $\mathrm{C}$ e $\mathrm{N}$ no solo, com implicações importantes para o balanço destes elementos em escala regional e global e para a produção sustentável e a qualidade ambiental. Esses autores destacam ainda que, além de melhorar a qualidade do solo, o uso de leguminosas resulta em menor custo financeiro e energético da produção de alimentos, pelo suprimento parcial do $\mathrm{N}$ e redução da necessidade de fertilizantes nitrogenados.

\section{Emissões médias mensais de $\mathrm{C}-\mathrm{CO}_{2}$}

As emissões de C-CO $\mathrm{CO}_{2}$ apresentaram padrão de variação temporal semelhante entre os sistemas de manejo (Figura 1b), sendo decrescente do verão de 2002/2003 até o inverno seguinte, crescente da primavera de 2003 até o verão de 2003/2004 e decrescente a partir de então. Nos meses de novembro e dezembro de 2002, as taxas de emissão de $\mathrm{C}-\mathrm{CO}_{2}$ do 
(a)

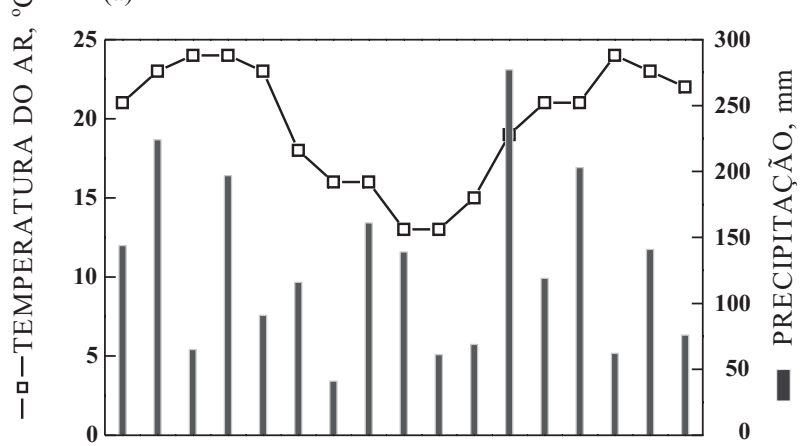

(b)

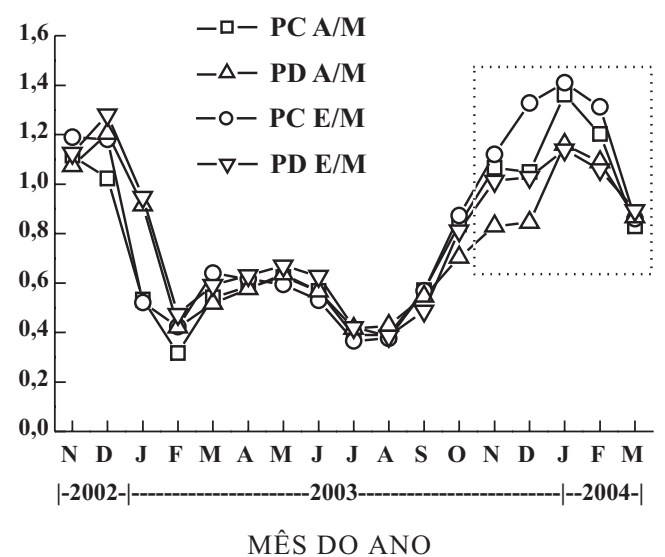

Figura 1. Médias mensais da temperatura do ar, da precipitação pluviométrica (a) e da emissão de $\mathrm{C}-\mathrm{CO}_{2}$ do solo (b) nas combinações de sistemas de preparo e de culturas. A área pontilhada representa o período logo após o manejo das culturas de inverno e realização dos preparos do solo, em outubro de 2003. PC: preparo convencional, PD: plantio direto, A: aveia-preta, E: ervilhaca comum e M: milho. solo dos sistemas variaram de 1,0 a 1,3 $\mathrm{g} \mathrm{m}^{-2}$, valores estes 25 e $62 \%$ maiores do que a média geral do período de avaliação $\left(0,8 \mathrm{~g} \mathrm{~m}^{-2}\right)$, respectivamente. Mesmo com temperaturas acima de $20^{\circ} \mathrm{C}$, a emissão de $\mathrm{C}-\mathrm{CO}_{2}$ em fevereiro de 2003 foi aproximadamente $50 \%$ menor do que a observada para o mesmo mês em 2004 (Figura 1b), devido principalmente à ocorrência de chuvas de até $80 \mathrm{~mm}$ concentradas neste mês, o que pode ter reduzido a atividade microbiana pela diminuição da concentração de $\mathrm{O}_{2}$ no solo. No período de outono/inverno de 2003, as taxas de emissão de C$\mathrm{CO}_{2}$ do solo, na média dos sistemas de manejo, foram $36 \%$ menores do que a média geral. Na primavera/ verão seguinte, as taxas de emissão de C-CO $\mathrm{CO}_{2}$ tiveram sua maior amplitude em relação à média geral, com valores de $0,5 \mathrm{~g} \mathrm{~m}^{-2}$ em setembro de 2003 a $1,4 \mathrm{~g} \mathrm{~m}^{-2}$ em janeiro de 2004 , as quais foram, respectivamente, $38 \%$ menor e $75 \%$ maior do que a média geral do período avaliado (Figura 1b).

As emissões mensais médias de $\mathrm{C}-\mathrm{CO}_{2}$ não diferiram significativamente entre os sistemas de manejo (Figura 1b). As médias das emissões do período total avaliado foram de $0,76( \pm 0,3)$ no $\mathrm{PC} \mathrm{A} / \mathrm{M}, 0,84$ $( \pm 0,4)$ no PC E/M, 0,76 ( $\pm 0,3)$ no PD A/M e 0,82 ( $\pm 0,3)$ $\mathrm{g} \mathrm{m}^{-2}$ de $\mathrm{C}-\mathrm{CO}_{2}$ no PD E/M, estando na faixa citada na literatura internacional (Duiker \& Lal, 2000). Contudo, após rolagem das culturas de inverno e, principalmente, após o preparo do solo, em outubro de 2003 (Figura 1b), embora sem diferença significativa, o solo em PC apresentou tendência de maior emissão de $\mathrm{C}-\mathrm{CO}_{2}$ do que em $\mathrm{PD}$, independentemente do sistema de cultura. A mesma tendência aconteceu com o sistema $\mathrm{E} / \mathrm{M}$ em relação ao $\mathrm{A} / \mathrm{M}$, sobretudo no solo em PC, onde o contato solo/resíduo foi aumentado pelo revolvimento do solo, permitindo a rápida decomposição dos tecidos das plantas de cobertura, especialmente da ervilhaca, que apresenta menor relação $\mathrm{C} / \mathrm{N}$.

Quadro 1. Estoque de C orgânico na camada de 0-0,2 m do solo em 1985 (início do experimento) e em 2003 (18 anos após) e sua variação anual, em relação a 1985, nas combinações dos sistemas de preparo de solo e de culturas

\begin{tabular}{|c|c|c|c|c|}
\hline \multirow{2}{*}{ Sistema de manejo } & \multicolumn{2}{|c|}{ Estoque } & \multirow{2}{*}{\multicolumn{2}{|c|}{ Variação no período ${ }^{(2)}$}} \\
\hline & 1985 & $2003^{(1)}$ & & \\
\hline & \multicolumn{2}{|c|}{$\mathrm{tha}^{-1}$} & $\mathrm{t} \mathrm{ha}^{-1}$ & $\mathrm{t} \mathrm{ha}^{-1} \mathrm{ano}^{-1}$ \\
\hline $\mathrm{PC}-\mathrm{A} / \mathrm{M}$ & 33,4 & $27,8 \mathrm{Bb}$ & $-5,6$ & $-0,31$ \\
\hline $\mathrm{PC}-\mathrm{E} / \mathrm{M}$ & 33,4 & $31,6 \mathrm{Ba}$ & $-1,8$ & $-0,10$ \\
\hline $\mathrm{PD}-\mathrm{A} / \mathrm{M}$ & 33,4 & $31,2 \mathrm{Ab}$ & $-2,2$ & $-0,12$ \\
\hline $\mathrm{PD}-\mathrm{E} / \mathrm{M}$ & 33,4 & $36,1 \mathrm{Aa}$ & 2,7 & 0,15 \\
\hline $\mathrm{CV}(\%)$ & - & 4,0 & - & - \\
\hline
\end{tabular}

${ }^{(1)}$ Preparos de solo seguidos de letras maiúsculas iguais, dentro de cada sistema de cultura, e sistemas de cultura seguidos de letras minúsculas iguais, dentro de cada preparo de solo, não diferem pelo teste de Duncan a $5 \%$. ${ }^{(2)}$ Sinais negativo e positivo significam emissão e retenção líquida de C no sistema solo-atmosfera, respectivamente. PD: plantio direto, PC: preparo convencional, A: aveia-preta, E: ervilhaca comum e M: milho. 
Os preparos do solo, em combinação com outras práticas de manejo e com a ação da temperatura e umidade do solo, influenciam a taxa de emissão de C$\mathrm{CO}_{2}$ para a atmosfera (Franzluebbers et al., 1995; Kessavalou et al., 1998), atuando sobre fatores que estão direta ou indiretamente relacionados à atividade microbiana, da qual depende a produção de $\mathrm{C}-\mathrm{CO}_{2}$. Os preparos de solo afetam sua cobertura e estrutura, as quais têm implicações na temperatura e umidade do solo e sobre os processos de trocas gasosas entre este e a atmosfera, além do aumento da disponibilidade de $\mathrm{C}$ lábil decorrente da incorporação de resíduos vegetais ao solo e exposição de matéria orgânica protegida no interior de agregados de solo (La Scala Jr., et al., 2005). Quanto ao efeito do tipo de resíduos adicionados ao solo, a relação $\mathrm{C}: \mathrm{N}$ destes parece ter influenciado as emissões de $\mathrm{C}-\mathrm{CO}_{2}$, sobretudo no período logo após a rolagem das culturas (outubro de 2003) no solo em PD. No solo em PC, o efeito foi pouco pronunciado logo após as operações de preparo, mas ficou evidente um mês após o revolvimento do solo. A menor relação C:N da ervilhaca $(15,4)$, em comparação à aveia-preta $(36,3)$, pode explicar a tendência de maior emissão de C-CO $\mathrm{CO}_{2}$ do solo sob a sucessão que contém essa leguminosa. Resíduos com relação $\mathrm{C}: \mathrm{N}$ baixa são mais facilmente decompostos pelos microrganismos do solo do que os com relação C:N alta (Tian et al., 1997). Por outro lado, no solo em PC, o revolvimento deste e o fracionamento dos resíduos vegetais podem ter auxiliado na liberação de $\mathrm{N}$ na fase inicial após os preparos, o que provavelmente colaborou para decomposição dos resíduos das culturas e para difusão do $\mathrm{C}-\mathrm{CO}_{2}$ à atmosfera.

\section{Relação da emissão de C-CO $\mathrm{CO}_{2}$ com a temperatura e umidade do solo}

A umidade gravimétrica do solo, nas três camadas amostradas, não foi afetada significativamente pelos sistemas de preparo e de cultura (dados não apresentados). O uso de sistema de irrigação durante o cultivo do milho e a boa distribuição e freqüência de chuvas no período, especialmente no inverno com baixas temperaturas do ar (Figura 1a) e do solo, podem estar associados a esse resultado. Contudo, na camada superficial (0 a $0,05 \mathrm{~m}$ ), embora o coeficiente de correlação entre a emissão de $\mathrm{C}-\mathrm{CO}_{2}$ e a umidade gravimétrica tenha sido baixo, a correlação negativa foi significativa (Figura 2a). A correlação entre a emissão de $\mathrm{C}-\mathrm{CO}_{2}$ e a umidade gravimétrica nas camadas de $0,05-0,1$ e de $0,1-0,2 \mathrm{~m}$ não foi significativa. E provável que esse resultado seja decorrente do fato de que as maiores umidades do solo tenham sido verificadas no inverno, devido à maior freqüência de chuva e menor evaporação (parcelas mantidas sem plantas).

Na média dos sistemas de manejo (sistemas de preparo + sistemas de culturas), a emissão de C-CO $\mathrm{CO}_{2}$ apresentou correlação com a temperatura do solo $(\mathrm{r}=0,88, \mathrm{p}<0,0001)$ (Figura $2 \mathrm{~b})$. No entanto, o coeficiente de correlação, na média dos sistemas de culturas, foi maior para o sistema PC $(r=0,91)$ do que para o $\mathrm{PD}(\mathrm{r}=0,85)$, indicando que houve relação mais direta da emissão de $\mathrm{C}-\mathrm{CO}_{2}$ em relação à temperatura do solo em $\mathrm{PC}$ e que outros fatores, além da temperatura do solo, podem estar influenciando a emissão de C-CO $\mathrm{CO}_{2}$ do solo em PD (Figura 2b). Nesse sentido, na média dos sistemas de cultura, o aumento (a)

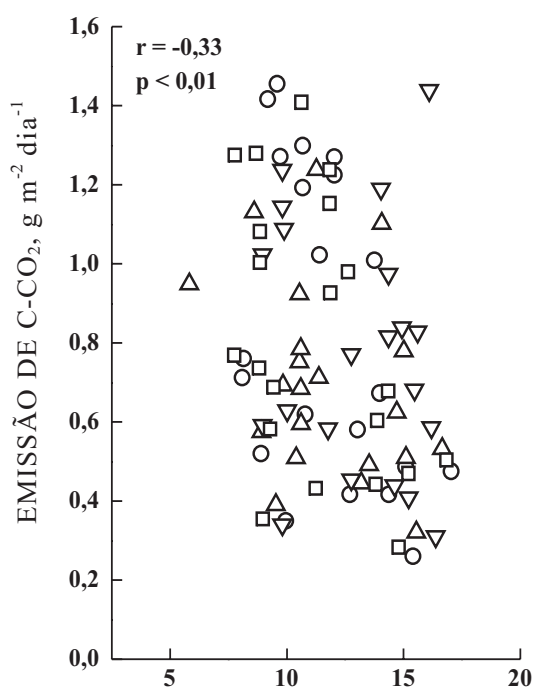

UMIDADE GRAVIMÉTRICA, 0-0,05 m (\%) (b)

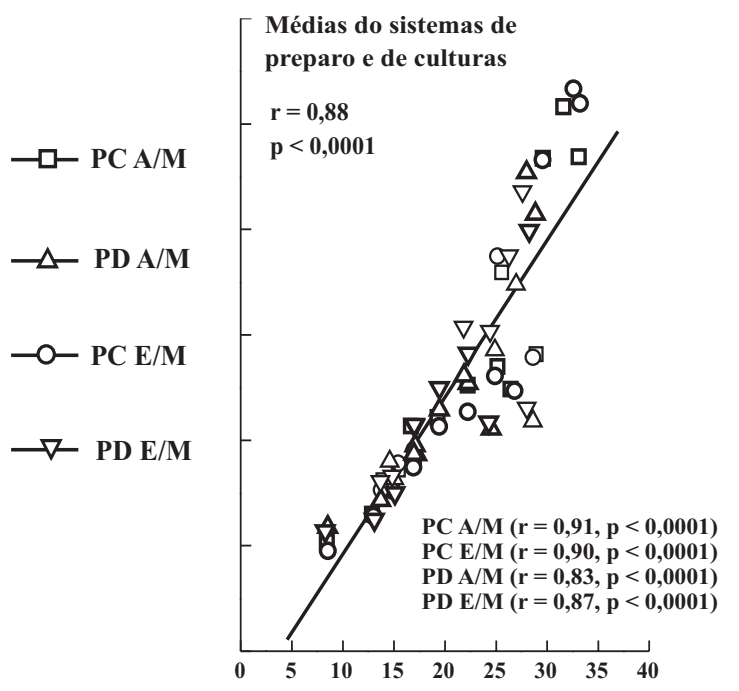

TEMPERATURA DIÁRIA MÉDIA, $0-0,05 \mathrm{~m}\left({ }^{\circ} \mathrm{C}\right)$

Figura 2. Emissão de $\mathrm{C}-\mathrm{CO}_{2}$ do solo em função da umidade gravimétrica (a) e da temperatura do solo (b), ambas na camada de 0-0,05 m do solo, no período de maio de 2003 a março de 2004. PC: preparo convencional, PD: plantio direto, A: aveia-preta, E: ervilhaca comum e M: milho. 
na taxa de emissão de C-CO $\mathrm{CO}_{2}$ em função do acréscimo de um grau na temperatura do solo $(0-0,05 \mathrm{~cm})$ foi reduzido em $20 \%$ pelo $\mathrm{PD}$, em relação ao PC. Quanto maior é a temperatura, menor é a energia de ativação que deve ser atingida para que uma reação aconteça (Paul \& Clark, 1996). Nesse caso, a ausência de cobertura do solo em PC, ao permitir incidência direta de raios solares, aumentou a temperatura do solo, que, por sua vez, intensificou a atividade microbiana, demonstrada pelo aumento da emissão de $\mathrm{C}-\mathrm{CO}_{2}$ do solo para a atmosfera (Figura $1 b$ ).

A correlação múltipla entre a emissão de $\mathrm{C}-\mathrm{CO}_{2}$ do solo, a temperatura média diária e a umidade gravimétrica do solo ( 0 a $0,05 \mathrm{~m})$ não foi significativa para inclusão deste último fator no modelo de análise.

\section{Efluxo total de $\mathrm{C}-\mathrm{CO}_{2}$ e capacidade dos sistemas de manejo em conservar $\mathrm{C}$ no solo}

As emissões totais de $\mathrm{C}-\mathrm{CO}_{2}$ variaram de 3,6 a 4,0 $\mathrm{t} \mathrm{ha}^{-1}$ e não diferiram significativamente entre os sistemas de manejo (Figura 3a). Esse comportamento similar das emissões totais entre os sistemas de manejo tem sido verificado por outros autores (Vargas \& Sholles, 2000), o que leva à seguinte questão: Como sistemas de manejo com taxas de emissões de $\mathrm{C}-\mathrm{CO}_{2}$ semelhantes apresentam diferentes acúmulos de $\mathrm{C}$ no solo? Essa aparente contradição ocorre porque a análise isolada das emissões de C-CO $\mathrm{CO}_{2}$ não é adequada para interpretar o potencial de sistemas de manejo em conservar ou acumular $\mathrm{C}$ no solo. Em comparação ao PC, no PD a maior proteção da matéria orgânica no interior dos agregados, o menor fracionamento dos resíduos e a menor área de contato dos resíduos com o solo reduzem a taxa de mineralização da matéria orgânica no solo, resultando em maior estoque de C. Outros fatores que se somam aos diferentes estoques de $\mathrm{C}$ orgânico no solo são a adição de quantidades diferenciadas de $\mathrm{C}$ via resíduo das culturas e sua relação $\mathrm{C} / \mathrm{N}$, os quais irão contribuir distintamente para o potencial de um sistema em emitir $\mathrm{C}-\mathrm{CO}_{2}$ à atmosfera.

Com o objetivo de avaliar o potencial dos sistemas de manejo em conservar $\mathrm{C}$ no solo, foi calculado o quociente entre as emissões de $\mathrm{C}-\mathrm{CO}_{2}$ e a quantidade de $\mathrm{C}$ no solo e nos resíduos vegetais, e o resultado, proposto como um índice de conservação de C (ICC) (Figura 3b). O ICC variou de $112 \pm 6,0 \mathrm{~g} \mathrm{~kg}^{-1}$ no sistema $\mathrm{PC} \mathrm{A} / \mathrm{M}$ a $90 \pm 4,0 \mathrm{~g} \mathrm{~kg}^{-1}$ no sistema PD E/M, sendo esses valores inversamente relacionados ao seu potencial de conservação de $\mathrm{C}$ no solo. $\mathrm{O}$ potencial de conservação de $\mathrm{C}$ no solo variou na seguinte ordem: $\mathrm{PD} \mathrm{E} / \mathrm{M}>\mathrm{PD} \mathrm{A} / \mathrm{M}>\mathrm{PC} \mathrm{E} / \mathrm{M}>\mathrm{PC} \mathrm{A} / \mathrm{M}$, reforçando a importância do $\mathrm{PD}$ na retenção do $\mathrm{C}$ no solo $\mathrm{e}$ destacando o papel das leguminosas no acúmulo de $\mathrm{C}$. $\mathrm{O} P \mathrm{PD}$ reduziu as taxas de emissão de $\mathrm{C}-\mathrm{CO}_{2}$, e a leguminosa contribuiu para aumento das quantidades de $\mathrm{C}$ aportado ao solo, via maior produção de resíduos de gramíneas cultivadas em sucessão, o que determinou a redução do ICC no PD dentro dos sistemas de culturas e no sistema E/M dentro dos sistemas de preparo.

A importância das leguminosas para o acúmulo de $\mathrm{N}$ no solo pode ser verificada quando se considera que a matéria orgânica humificada do solo apresenta relação C:N de aproximadamente 12:1 (Amado et al., 2001; Lovato et al., 2004). Nessa condição, estima-se que para acumular 1,0 t de C é necessário acumular aproximadamente $0,08 \mathrm{t}$ de $\mathrm{N}$. Ou seja, para acumular $\mathrm{C}$, é necessário acumular $\mathrm{N}$, e isso reforça a importância da inclusão de leguminosas em sistemas de sucessão ou rotação de culturas, além dos seus efeitos na redução de custos pelo suprimento parcial de $\mathrm{N}$ às culturas comerciais. Convém salientar que esse efeito positivo da adoção de leguminosas no acúmulo de $\mathrm{C}$ no solo é válido provavelmente só para leguminosas utilizadas para cobertura do solo, não sendo válido no caso da soja, cujo $\mathrm{N}$ fixado biologicamente sai do sistema quando da colheita dos grãos. Isso significa dizer que, mesmo em sistemas de produção de soja, é necessária a inclusão de leguminosas de cobertura de solo, e isso pode ser feito anteriormente à cultura do milho cultivado em rotação com a soja.

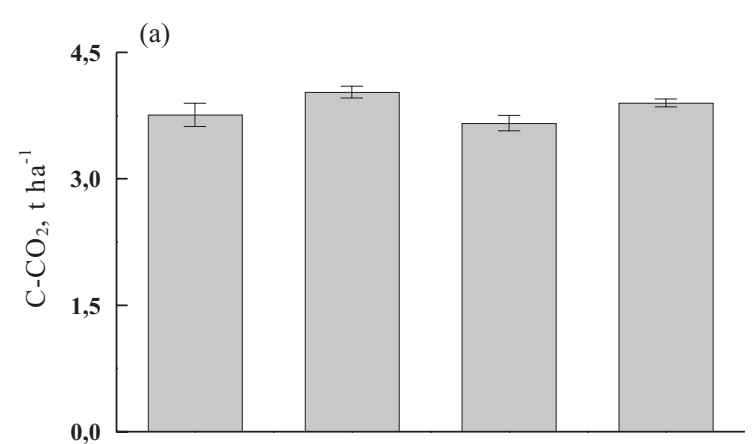

(b)

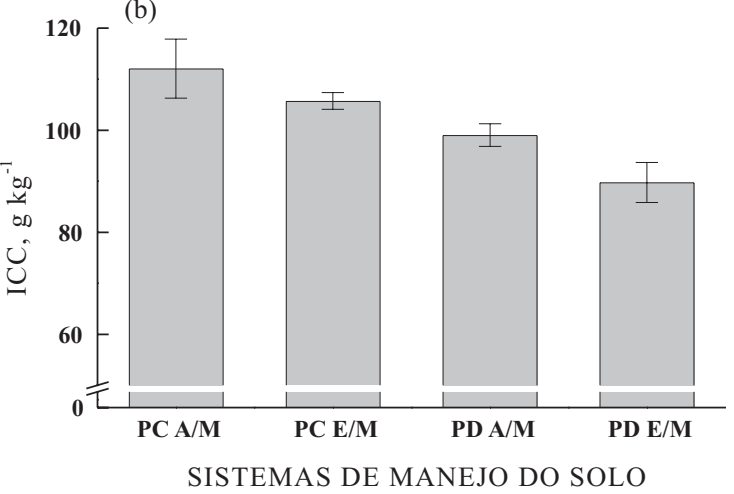

Figura 3. Emissão total de $\mathrm{C}-\mathrm{CO}_{2}$ do solo no período de 17 meses (a) e Índice de Conservação de Carbono-ICC (b), calculado pela razão entre a emissão total de $\mathrm{C}-\mathrm{CO}_{2}$ e a quantidade de $\mathrm{C}$ nos resíduos vegetais e na matéria orgânica no solo em um Argissolo Vermelho sob diferentes sistemas de preparo e de cultura. PC: preparo convencional, PD: plantio direto, A: aveia-preta, E: ervilhaca comum e M: milho. Barras verticais: erro-padrão da média. 


\section{CONCLUSÕES}

1. O plantio direto associado a sistemas de cultura com alta adição de resíduos vegetais ricos em $\mathrm{C}$ e N resulta em balanço positivo de $\mathrm{C}$ no solo.

2. O solo em preparo convencional, independentemente do sistema de cultura, e o solo em plantio direto associado a sistemas de cultura com baixo aporte de resíduos vegetais apresentam balanço negativo de C.

3. As emissões totais de $\mathrm{C}-\mathrm{CO}_{2}$ do solo são similares nos diferentes sistemas de manejo; entretanto, quando as emissões de $\mathrm{C}-\mathrm{CO}_{2}$ são ponderadas em função da quantidade de $\mathrm{C}$ no solo e nos resíduos vegetais, é possível verificar a importância do plantio direto e da inclusão de plantas de cobertura leguminosas na conservação do $\mathrm{C}$ no solo.

4. As emissões de $\mathrm{C}-\mathrm{CO}_{2}$ têm variação sazonal e são correlacionadas positivamente com a temperatura do solo.

\section{AGRADECIMENTOS}

Ao Professor Homero Bergamaschi (Departamento de Forrageiras e Agrometereologia da Faculdade de Agronomia da UFRGS), pelo fornecimento dos dados de temperatura e precipitação pluvial do período em que o trabalho foi realizado. À FAPERGS, ao CNPq e ao MCT, pelo apoio financeiro (Projeto Pronex 04/ 0850.0 - Seqüestro de carbono e mitigação das emissões de gases de efeito estufa por sistemas conservacionistas de manejo e as oportunidades para o agronegócio no $\mathrm{RS}$ ”).

\section{LITERATURA CITADA}

AITA, C.; CERETTA, C.A.; THOMAS, A.L.; PAVINATO, A. \& BAYER, C. Espécies de inverno como fonte de nitrogênio para o milho no sistema de cultivo mínimo e feijão em plantio direto. R. Bras. Ci. Solo, 18:101-108, 1994.

AMADO, T.J.C.; BAYER, C.; ELTZ, F.L.F. \& BRUM, A.C.R. Potencial de culturas de cobertura em acumular carbono e nitrogênio no solo no plantio direto e a melhoria da qualidade ambiental. R. Bras. Ci. Solo, 25:189-197, 2001.

ANDERSON, J.P.E. Soil respiration. In: PAGE, A.L.; MILLER, R.H. \& KEENEY, D.R., eds. Methods of soil analysis. Part 2 - Chemical and microbiological properties 2.ed. Madison, Soil Science Society of America, 1982. p.837-871. (Agronomy Monograph, 9)

BALESDENT, J. \& BALABANE, M. Maize root-derived soil organic carbon estimated by natural 13C abundance. Soil Biol. Biochem., 24:97-101, 1992.

BAYER, C.; MIELNICZUK, J. \& PAVINATO, A. Sistemas de manejo do solo e seus efeitos sobre o rendimento do milho. Ci. Rural, 28:23-28, 1998 .
BAYER, C.; MARTIN-NETO, L.; MIELNICZUK, J.; PAVINATO, A. \& DIECKOW, J. Carbon sequestration in two Brazilian Cerrado soils under no-till. Soil Till. Res., $86: 237-245,2006$

BAYER, C.; MIELNICZUK, J. \& MARTIN-NETO, L. Efeito de sistemas de preparo e de cultura na dinâmica da matéria orgânica e na mitigação das emissões de $\mathrm{CO}_{2}$. R. Bras. Ci. Solo, 24:599-607, 2000.

BERGAMASCHI, H.; GUADAGNIN, M.R.; CARDOSO, L.S. \& SILVA, M.I.G. Clima da Estação Experimental da UFRGS (e Região de Abrangência). Porto Alegre, Universidade Federal do Rio Grande do Sul, 2003. 78p.

BOLINDER, M.A.; ANGERS, D.A. \& DUBUC, J.P. Estimating shoot to root ratios and annual carbon inputs in soil for cereal crops. Agric. Ecosyst. Environ., 63:61-66, 1997.

BUYANOVSKY, G.A. \& WAGNER, G.H. Post-harvest residue input to cropland. Plant Soil, 93:57-65, 1986.

CIOTTA, M.N.; BAYER, C.; ERNANI, P.R.; FONTOURA, S.M.V.; WOBETO, C. \& ALBUQUERQUE, J.A. Manejo da calagem e seu efeito sobre componentes da acidez de um Oxisol sob plantio direto. R. Bras. Ci. Solo, 27:527-535, 2004.

COSTA, F.S.; GOMES, J.; BAYER, C. \& MIELNICZUK, J. Métodos para avaliação das emissões de gases de efeito estufa no sistema solo-atmosfera. Ci. Rural, 36:693-700, 2006.

COSTA, F.S.; ALBUQUERQUE, J.A.; BAYER, C.; FONTOURA, S.M.V. \& WOBETO, C. Propriedades físicas de um Latossolo Bruno afetadas pelos sistemas de plantio direto e preparo convencional. R. Bras. Ci. Solo, 27:527535, 2003.

CROZIER, C.R. \& KING, L.D. Corn root dry matter and nitrogen distribution as determined by sampling multiple soil cores around individual plants. Comm. Soil Sci. Plant Anal., 24:1127-1138, 1993.

DRINKWATER, L.E.; WAGONER, P. \& SARRANTONIO, M. Legume-based cropping systems have reduced carbon and nitrogen losses. Nature, 396:262-265, 1998.

DUIKER, S.W. \& LAL, R. Carbon budget study using $\mathrm{CO}_{2}$ flux measurements from a no till system in central Ohio. Soil Till. Res., 54:21-30, 2000.

ELLERT, B.H. \& BETTANY, J.R. Calculation of organic matter and nutrients stored in soils under contrasting management regimes. Can. J. Soil Sci., 75:529-538, 1995.

EMPRESA BRASILEIRA DE PESQUISA AGROPECUÁRIA EMBRAPA. Centro Nacional de Pesquisa de Solos. Sistema brasileiro de classificação de solos. Brasília, Embrapa Produção de Informação, 1999. 412p.

FEHRENBACHER, J.B. \& ALEXANDER, J.D. A method for studying corn root distribution using a soil-core sampling machine and shakertype washer. Agron. J., 47:468-472, 1955.

FRANZLUEBBERS, A.J.; HONS, F.M. \& ZUBERER, D.A. Tillage-induced seasonal changes in soil physical properties affecting soil $\mathrm{CO}_{2}$ evolution under intensive cropping. Soil Till. Res., 34:41-60, 1995. 
INTERGOVERNMENTAL PANEL ON CLIMATE CHANGE - IPCC. Climate change 2001: The scientific basis. Cambridge, Cambridge University Press, 2001. 881p.

JANZEN, H.H.; CAMPBELL, C.A.; IZAURRALDE, R.C.; ELLERT, B.H.; JUMA, N.; McGILL, W.B. \& ZENTNER, R.P. Management effects on soil C storage on the Canadian prairies. Soil Till. Res., 47:181-195, 1998.

KESSAVALOU, A.; DORAN, J.W.; MOSIER, A.R. \& DRIJBER, R.A. Greenhouse gas fluxes following tillage and wetting in a wheat-fallow cropping system. J. Environ. Qual., 27:1105-1116, 1998.

KISSELE, K.W.; GARRETT, C.J.; FU, S.; HEDRIX, P.F.; CROSSLEY, D.A.; COLEMAN, D.C. \& POTTER, R.L. Budgets for roots-derived $\mathrm{C}$ and litter-derived $\mathrm{C}$ : Comparison between conventional tillage and no tillage soils. Soil Biol. Biochem., 33:1067-1075, 2001.

LA SCALA Jr., N.; BOLONHEZI, D. \& PEREIRA, G.T. Shortterm soil $\mathrm{CO}_{2}$ emission after conventional and reduced tillage of a no till sugar cane area in Southern Brazil. Soil Till. Res., 91:244-248, 2006.

LA SCALA Jr., N.; LOPES, A.; PANOSSO, A.R.; CAMARA, F.T. \& PEREIRA, G.T. Soil $\mathrm{CO}_{2}$ efflux following rotary tillage of a tropical soil. Soil Till. Res., 84:233-235, 2005.

LOVATO, T. Dinâmica do carbono e nitrogênio do solo afetada por preparos do solo, sistemas de cultura e adubo nitrogenado. Porto Alegre, Universidade Federal do Rio Grande do Sul, 2001. 133p. (Tese de Doutorado)

LOVATO, T.; MIELNICZUK, J.; BAYER, C. \& VEZZANI, F. Adições de carbono e nitrogênio e sua relação com os estoques no solo e com o rendimento do milho em sistemas de manejo. R. Bras. Ci. Solo, 28:175-187, 2004.
PAUL, E.A. \& CLARK, F.E. Soil microbiology and biochemistry. 2.ed. San Diego, Academic Press, 1996. 340p.

PAUSTIAN, K.; ANDRÉN, O.; JANZEN, H.H.; LAL, R.; SMITH, P.; TIAN, G.; TIESSEN, H.; Van NOORDWIJK, M. \& WOOMER, P.L. Agricultural soils as a sink to mitigate $\mathrm{CO}_{2}$ emissions. Soil Use Manag., 13:230-244, 1997.

SILVA, M.A.S.; MAFRA, A.L.; ALBUQUERQUE, J.A.; BAYER, C. \& MIELNICZUK, J. Atributos físicos do solo relacionados ao armazenamento de água em um Argissolo Vermelho sob diferentes sistemas de preparo. Ci. Rural, 35:544-552, 2005 .

SPAGNOLLO, E.; BAYER, C.; WILDNER, L.P.; ERNANI, P.R.; ALBUQUERQUE, J.A. \& PROENÇA, M.M. Leguminosas estivais intercalares como fonte de nitrogênio para o milho, no Sul do Brasil. R. Bras. Ci. Solo, 26:417-423, 2002 .

TIAN, G.; BRUSSAARD, L.; KANG, B.T. \& SWIFT, M.J. Soil fauna-mediated decomposition of plants residues under constrained environmental and residue quality conditions. In: CADISCH, G. \& GILLER, K.E. eds. Driven by nature: Plant and litter quality and decomposition. Wallingford, CAB International, 1997. p.125-134.

VARGAS, L.K. \& SCHOLLES, D. Biomassa microbiana e produção de $\mathrm{C}-\mathrm{CO}_{2}$ e $\mathrm{N}$ mineral de um Podzólico Vermelho-Escuro submetido a diferentes sistemas de manejo. R. Bras. Ci. Solo, 24:35-42, 2000.

ZANATTA, J.A.; BAYER, C.; DIECKOW, J.; VIEIRA, F.C.B. \& MIELNICZUK, J. Soil organic carbon accumulation and carbon costs related to tillage, cropping systems and nitrogen fertilization in a subtropical Acrisol. Soil Till. Res., 94:510-519, 2007. 